\title{
EBDHR: Energy Balancing and Dynamic Hierarchical Routing algorithm for wireless sensor networks
}

\author{
Saeed Rasouli Heikalabad ${ }^{\text {a)}}$, Ahmad Habibizad Navin, Mirkamal \\ Mirnia, Saeed Ebadi, and Mehdi Golesorkhtabar \\ Computer Research Lab, Islamic Azad University-Tabriz Branch, Tabriz, Iran \\ a)s.rasouli.h@gmail.com
}

\begin{abstract}
Due to energy resources constraint in wireless sensor networks, balancing energy consumption is very important in increasing the network life time. In this paper, the new hierarchical and dynamic routing algorithm is proposed to balance energy consumption among the nodes and to prevent from energy holing problem. Simulation results show that the proposed algorithm prolongs the network lifetime about $40 \%$ compared to the LEACH protocol.
\end{abstract}

Keywords: wireless sensor networks, dynamic routing, hierarchical algorithm, energy balancing

Classification: Science and engineering for electronics

\section{References}

[1] D. Estrin, L. Girod, G. Pottie, and M. Srivastava, "Instrumenting the World with Wireless Sensor Networks," IEEE Int. Conf. Acoustics, Speech, and Signal Process., vol. 4, pp. 2033-2036, 2001.

[2] L. F. Akyildiz, S. Weilian, Y. Sankarasubramaniam, and E. Cayirci, "A Survey on Sensor Networks," IEEE Commun. Mag., vol. 40, no. 8, pp. 102-114, 2002.

[3] M. Ettus, "System Capacity, Latency, and Power Consumption in Multihop-routed SS-CDMA Wireless Networks," Radio and Wireless Conference, pp. 55-58, 1998.

[4] W. B. Heinzelman, A. P. Chandrakasan, and H. Balakrishnan, "An Application-Specific Protocol Architecture for Wireless Microsensor Networks," IEEE Trans. Wireless Commun., vol. 1, no. 4, 2002.

[5] W. B. Heinzelman, A. P. Chandrakasan, and H. Balakrishnan, "EnergyEfficient Communication Protocol for Wireless Microsensor Networks," Proc. Hawaii Int. Conf. System Sciences, 2000.

[6] O. Younis and S. Fahmy, "Distributed Clustering in Ad-hoc Sensor Networks: A Hybrid, Energy-Efficient Approach," Proc. IEEE INFOCOM, vol. 1, pp. 629-640, 2004.

[7] O. Younis and S. Fahmy, "HEED: A Hybrid, Energy-Efficient, Distributed Clustering Approach for Ad Hoc Sensor Networks," IEEE Trans. Mobile Computing, vol. 3, no. 4, pp. 366-379, 2004.

[8] D. Xia and N. Vlajic, "Near-optimal Node Clustering in Wireless Sensor Network for Environment Monitoring," 21st Int. Conf. Advanced Networking and Applications, pp. 632-641, 2007. 
[9] M. S. Kordafshari, A. Pourkabirian, K. Faez, and A. M. Rahimabadi, "Energy-Efficient SPEED Routing Protocol for Wireless Sensor Networks," Fifth Advanced International Conference on Telecommunications, pp. 267-271, 2009.

[10] I. Shin, M. Kim, M. W. Mutka, H. Choo, and T. J. Lee, "MCBT: MultiHop Cluster Based Stable Backbone Trees for Data Collection and Dissemination in WSNs," MDPI Sensors, vol. 9, pp. 6028-6045, 2009.

[11] Q. Shi, H. Huo, T. Fang, and D. Li, "A 3D Node Localization Scheme for Wireless Sensor Networks," IEICE Electron. Express, vol. 6, no. 3, pp. 167-172, 2009.

\section{Introduction}

Recent advances in WSN's technology, use of these networks significantly increased in various fields including monitoring, commercial, military and industrial [1]. Wireless sensor networks are composed of very small nodes which are able to sense the information, perform some processes and create wireless communications between each other or directly with base station (BS) or sink. These networks have very constrain resources such as memory, power and process units and an energy constrain problem is more important among them [2].

Several routing protocols such as directed transmission (DT), minimum transmission energy (MTE) [3] and clustering protocols [4-6] have been proposed for saving the energy and lifetime increasing. In DT, all nodes transmit data to the base station directly while in MTE, nodes send the data to the destination in multi hop. Since there is unbalancing energy consumption problem in both methods, thus energy of the nodes far from the base station in DT and also the energy of the nodes close to the base station in MTE discharge faster than the rest. In compares on with previous two protocols, clustering protocol increases the network efficiency [4]. The data of all nodes within clusters in this protocol are collected by cluster heads and are transmitted to the base station after fusion. Communication between cluster heads can be direct or multi hop. Therefore unbalancing energy consumption problem among the cluster heads exists yet [6].

In wireless sensor networks, one of the most famous cluster based routing protocols is LEACH [5]. In this protocol, the cluster heads are communicated directly with base station which causes the unbalanced energy consumption between them and therefore energy source of cluster heads far from the base station are discharged earlier than the others.

\section{Related works}

HEED [7] selects cluster-heads according to a combination of the sensor residual energy and a secondary parameter such as node proximity to its neighbors or degree of node. LNCA [8] introduces a novel clustering algorithm which uses the similarity of sensed data as an important factor in cluster formation. 
In EE_SPEED [9], routing is based on a weight function, which is a combination of the three factors: Delay, Energy and Speed. MCBT [10] proposes a distributed algorithm to create a stable backbone by selecting the nodes with higher energy or degree as the cluster heads. LEACH [5] is containing of four phases that are the advertisement, cluster formation, scheduler creation and data transmission. In first phase, nodes compete with each other for election as cluster head so that all nodes produce a random number between 0 and 1 and then produced numbers should be compared with threshold value which is obtained by Eq. (1). If produced number is smaller than $T(n)$, then the node is selected as a cluster head.

$$
T(n)= \begin{cases}\frac{p}{1-p(r \bmod (1 / p))} & n \in G \\ 0 & \text { others }\end{cases}
$$

In here $p$ is percent of cluster heads to all nodes, $r$ is current round and $G$ is the set of nodes that are not selected as cluster head in $1 / p$ of last rounds.

In the second phase, nodes are joined to their near cluster heads and form the clusters. In the next phase, cluster heads create the TDMA scheduler. In the last phase, all nodes transmit the data to their cluster heads based on the created scheduler.

\section{Proposed protocol}

In EBDHR, election of cluster heads and cluster formation is like LEACH and the main difference is in data transmission from cluster heads to the base station. This operation in the LEACH is done directly, but in the EBDHR it is hierarchical and dynamic. The proposed algorithm consider the distance between cluster heads and base station in multi hop in most of times and hence eliminates the unbalancing energy consumption problem and prevents the rapid energy consumption of cluster heads near base station unlike the other hierarchical routing protocols.

\subsection{Definition}

A cluster head is a high level one (HLCH) if its distance to base station is less than the distance of sender cluster head to base station.

\subsection{Assumptions}

- All nodes are randomly distributed.

- Nodes are static or pseudo static.

- The initial energy is the same for all nodes.

- Nodes are aware of the location (by positioning schemes such as [11]).

- Nodes are able to control their energy consumption.

- Base station is aware from remaining energy of cluster heads (via received beacon from the cluster heads when the level of their energy is going to be changed). 
- Cluster heads are aware from their remaining energy and also from the remained energy of their HLCHs (via received beacon from the base station).

- Base station is in the center of operation field.

\subsection{Energy consumption model}

In EBDHR, energy model is obtained from [5] that uses both of the open space (energy dissipation $d^{2}$ ) and multi path (energy dissipation $d^{4}$ ) channels by taking amount the distance between the transmitter and receiver. So energy consumption for transmitting a packet of $l$ bits in distance $d$ is given by

$$
E_{T x}(l, d)=\left\{\begin{array}{l}
l E_{\text {elec }}+l \varepsilon_{f s} d^{2}, \mathrm{~d} \leq \mathrm{d}_{0} \\
l E_{\text {elec }}+l \varepsilon_{m p} d^{4}, \mathrm{~d}>\mathrm{d}_{0}
\end{array}\right.
$$

In here $d_{0}$ is the distance threshold value which is obtained by Eq. (3), $E_{\text {elec }}$ is required energy for activating the electronic circuits. $\varepsilon_{f s}$ and $\varepsilon_{m p}$ are required energy for amplification of transmitted signals to transmit a one bit in open space and multi path models, respectively.

$$
d_{0}=\sqrt{\frac{\varepsilon_{f s}}{\varepsilon_{m p}}} .
$$

Energy consumption to receive a packet of $l$ bits is calculated according to

$$
E_{R x}(l)=l E_{\text {elec }}
$$

\subsection{EBDHR}

The main contribution of proposed algorithm is to use the Eq. (5) and Eq. (6) for selecting the $\mathrm{HLCH}$ in dynamic to transfer the aggregated data to the base station.

$$
\begin{gathered}
\text { Confidence_Value } \_1=\frac{E_{L C H}}{\left(D_{L C H \_B S}\right)^{2}} . \\
\text { Confidence_Value_2 }=\frac{E_{L C H}+E_{H C H}}{\left(D_{\left.L C H_{-} H_{C H}+D_{H C H \_B S}\right)^{2}}\right.} .
\end{gathered}
$$

In here $E_{L C H}$ is the remaining energy of cluster head that transfers the data to the base station and $E_{H C H}$ is the remaining energy of HLCH. $D_{L C H_{-} B S}$ and $D_{L_{C H} H_{-} H_{C H}}$ are the distances between cluster heads that transmit the data to the base station and to the $\mathrm{HLCH}$ respectively. $D_{H_{C H} H_{-} S}$ is the distance between HLCH and base station.

The beginning, the cluster head which is the transmitter, calculates the own Confidence_Value by Eq. (5) and then obtains the Confidence_Value of HLCHs from Eq. (6). If Confidence_Value of each HLCHs is greater than the Confidence_Value of remaining HLCHs and transmitter, then desirable cluster head is selected as data transmitter to the base station. Then HLCH selection operation repeats for this cluster head until the data is transferred to the base station. When the Confidence_Value of transmitter cluster head is greatest, data is sent directly to the base station. The sample of these operations illustrates in Fig. 1. 


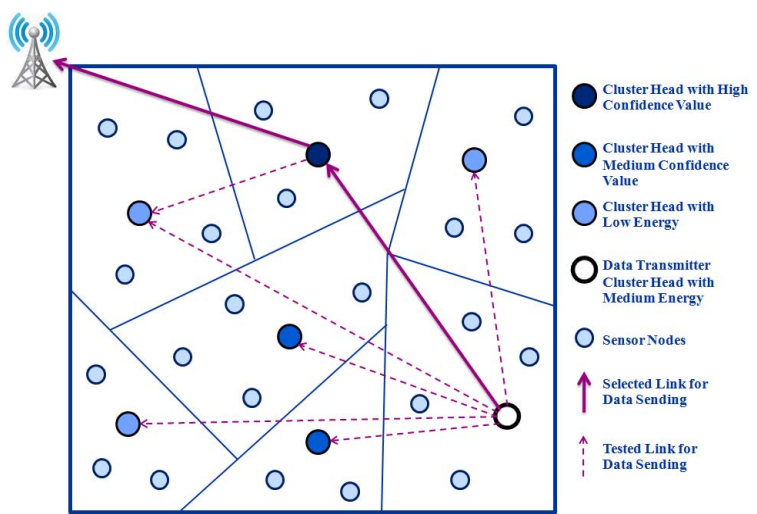

Fig. 1. Illustration of data transmitting operations in EBDHR.

In contrast to LEACH, cluster heads in EBDHR can use their HLCHs for data transmission. Thus energy consumption is balanced among the nodes and therefore the lifetime of network increases.

To guarantee to achieve the required quality of service of real time applications, in EBDHR, each of the cluster heads directly sends a beacon message to the base station to announce itself remaining energy when the level of its energy is going to be changed. Then base station is immediately broadcasts the received beacon message to the cluster heads located at the lower level of the cluster head that its energy has changed to announce the amount of its energy.

\section{Simulation and result analysis}

EBDHR and LEACH are simulated with GCC and the simulation repeated for two times with different simulation areas and number of nodes to achieve the reliable results about proposed algorithm. Simulation parameters are presented in Table I and obtained results are shown below.

Table I. Simulation parameters.

\begin{tabular}{l|l}
\hline Parameters & Value \\
\hline Simulation area & 200 meters $\times 200$ meters \\
\hline Base station location & $(100,100) \mathrm{m}$ \\
\hline $\mathrm{N}$ & 200 \\
\hline Initial energy & $3 \mathrm{~J}$ \\
\hline$E_{\text {elec }}$ & $50 \mathrm{~nJ} / \mathrm{bit}$ \\
\hline$\varepsilon_{f s}$ & $10 \mathrm{pJ} / \mathrm{bit} / \mathrm{m}^{2}$ \\
\hline$\varepsilon_{m p}$ & $0.0013 \mathrm{pJ} / \mathrm{bit} / \mathrm{m}^{4}$ \\
\hline$d_{0}$ & $87 \mathrm{~m}$ \\
\hline$E_{D A}$ & $5 \mathrm{~nJ} / \mathrm{bit} / \mathrm{signal}$ \\
\hline Data packet size & $8192 \mathrm{bits}$ \\
\hline Beacon message size & $40 \mathrm{bits}$ \\
\hline $\begin{array}{l}\text { The desired percentage to } \\
\text { become a cluster-head }\end{array}$ & $5 \%$ \\
\hline
\end{tabular}

Fig. 2 (a) and 2 (b) shows how the active and dead nodes are situated in EBDHR and LEACH in the round that half of the nodes are dead. 
As it can be seen, the nodes in EBDHR are died uniformly and independent of its location, but in LEACH, nodes away the base station are died with high probability and earlier than others that causes the energy holing problem. Moreover, in some rounds that energy of HLCHs is low EBDHR acts the same as LEACH and thus prevents fast energy consumption of the nodes close to the BS.

Fig. 2 (c) shows the residual energy of whole network per round for LEACH and EBDHR. As it can be seen, obtained values for EBDHR is better than LEACH.

Fig. 2 (d) and 2 (e) shows the number of dead nodes per round in LEACH

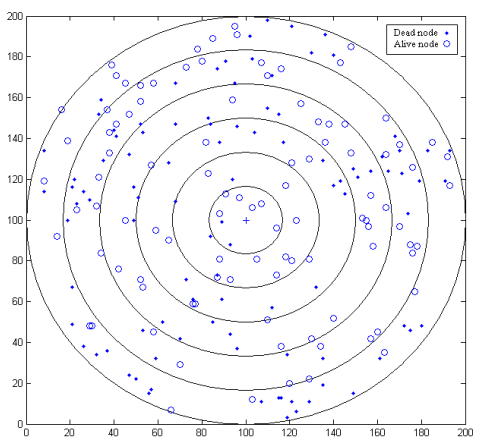

(a)

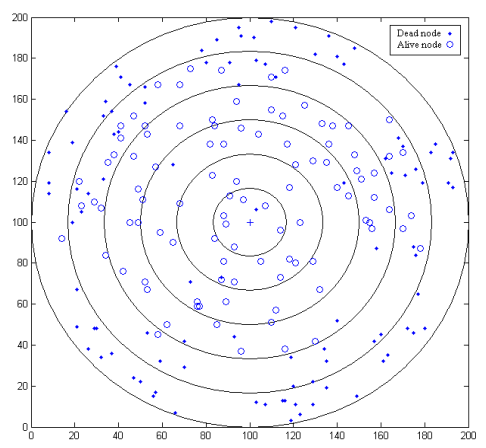

(b)

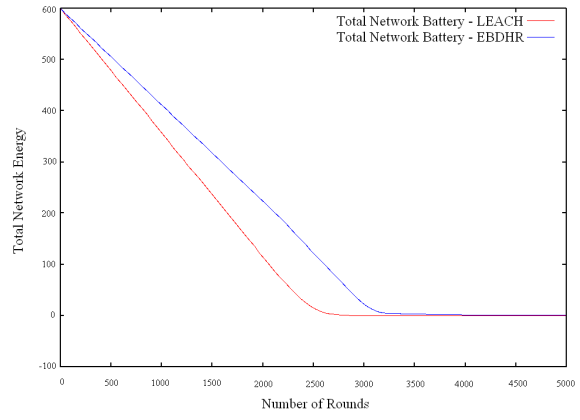

(c)

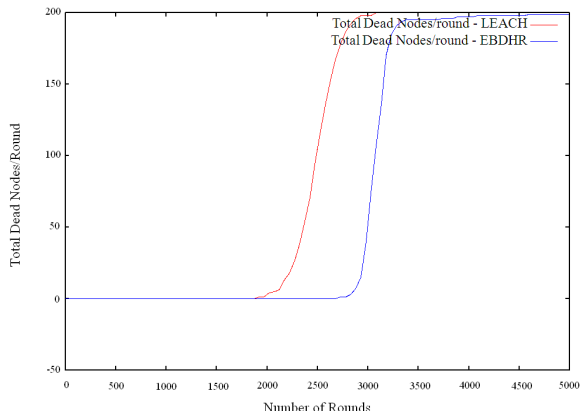

(d)

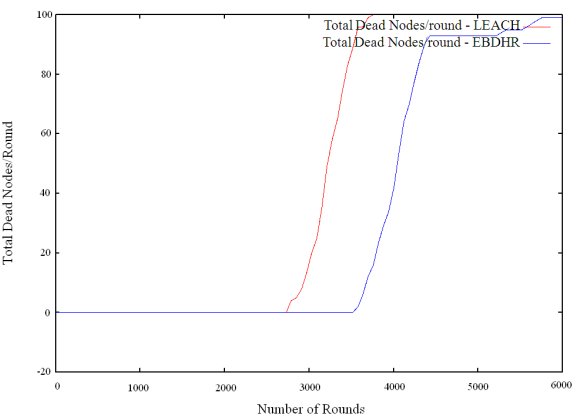

(e)

Fig. 2. Simulation results. (a) Locations of dead and active sensor nodes in EBDHR, (b) Locations of dead and active sensor nodes in LEACH, (c) Residual energy of whole network per round with default parameters, (d) number of dead nodes per round with default parameters, and (e) number of dead nodes per round with 100 nodes in $100 \mathrm{~m} * 100 \mathrm{~m}$ area. 
and EBDHR in the network with different nodes and areas.

As it can be seen, proposed protocol has a performance better than LEACH protocol so that in EBDHR, the times of first node dies (FND) and half nodes alive (HNA) and last node dies (LND) are optimized about $48 \%, 29 \%$ and $44 \%$, respectively.

\section{Conclusions and further work}

This work proposes a new routing algorithm for WSN namely EBDHR. The main contribution of this algorithm is to select high level cluster head by low level cluster head in dynamic based on both remained energies and distances from BS for balancing the energy consumption among nodes. Simulation results show that the EBDHR prolong the network lifetime about $40 \%$ in comparison to the LEACH.

Our research group is going to uses the proposed algorithm in inter-cluster to balancing the energy consumption among active nodes within the clusters.

\section{Acknowledgments}

The authors would like to thanks from Dr. Ali Ghaffari and Dr. Shahram Babaie. 